\title{
Down-Regulation of CD9 Expression and its Correlation to Tumor Progression in B Lymphomas
}

\author{
Sun-Ok Yoon, ${ }^{*}$ Xin Zhang, ${ }^{*}$ Arnold S. Freedman, ${ }^{\dagger}$ \\ David Zahrieh, ${ }^{\ddagger}$ Izidore S. Lossos, ${ }^{\S}$ Li Li, ${ }^{*}$ \\ and Yong Sung Choi* \\ From the Laboratory of Cellular Immunology,* Ochsner Clinic \\ Foundation, New Orleans, Louisiana; the Departments of \\ Medical Oncology, ${ }^{\dagger}$ and Biostatistics and Computational \\ Biology, ${ }^{\ddagger}$ The Dana-Farber Cancer Institute, Harvard Medical \\ School, Boston, Massachusetts; and the Department of Medicine, \\ Division of Hematology-Oncology and Department of Molecular \\ and Cellular Pharmacology, ${ }^{\S}$ University of Miami, Miami, Florida
}

Histological transformation, a pivotal event in the natural history of cancers including lymphomas, is typically associated with more aggressive clinical behavior. L3055, a B lymphoma cell line of germinal center (GC) origin, is dependent on follicular dendritic cells (FDCs) for survival and proliferation, similar to GC-B cells. However, L3055 cells become less FDC-dependent after prolonged culture, which is analogous to transformation in vivo. Comparison of two L3055 subclones (i.e., the FDC-dependent indolent clone 12 and the FDC-independent aggressive clone 33) by DNA microarray revealed that $C D 9$ was the most differentially expressed gene $(P=0.05)$. L3055-12 expresses high levels of CD9 while L3055-33 does not. Reduced levels or loss of CD9 expression is also observed in other CD9-positive B lymphoma cell lines. The resultant $C D$ 9-negative cells grow faster than CD9-positive cells due to their greater resistance to apoptosis. Furthermore, CD9-negative cells are less dependent on FDCs for their survival and growth compared with CD9-positive cells. CD9 down-regulation in $B$ lymphomas appears to be controlled epigenetically, mainly through histone modifications. These findings imply that CD9 is inversely correlated with B lymphoma progression, and CD9 inactivation may play an important role in B lymphoma transformation. (Am J Pathol 2010, 177:377-386; DOI: 10.2353/ajpath.2010.100048)

Most B cell lymphomas emerge in the germinal center (GC) of lymphoid tissues. ${ }^{1,2}$ The GC is a specialized anatomical microenvironment where Ag-activated B cells undergo clonal expansion and selection and differentiate into plasma cells and memory B cells. Somatic hypermutation of $\operatorname{lgV}_{\mathrm{H}}$ and isotype switching from IgM to other isotypes take place during GC-B cell proliferation. ${ }^{3,4}$ During the rapid cellular proliferation and genetic changes, genetic instability occurs, inducing malignant transformations of GC-B cells. Follicular dendritic cells (FDCs), which are major stromal cells localized in the $\mathrm{GC},{ }^{5,6}$ play critical roles in the organization of local microenvironment by forming reticular networks and provide a milieu of factors for survival and proliferation of GC-B cells. In the initial indolent stage, lymphoma cells are dependent on FDCs for their survival and proliferation similar to normal GC-B cells. ${ }^{7}$ However, indolent lymphoma cells eventually transform to become more aggressive lymphomas, which is a common pathway of virtually all tumor cells. The loss of FDCs within neoplastic follicles is considered to precede this transformation. ${ }^{8}$ Despite the advances in understanding the transformation of tumors, the mechanisms of transformation from indolent lymphomas to aggressive lymphomas under the influence of FDCs are poorly understood because of the lack of proper experimental models.

In this study, we have created an experimental model that mimics the transformation of $B$ lymphomas where indolent FDC-dependent lymphomas become aggressive FDC-independent lymphomas. A lymphoma cell line of the GC origin, L3055, requires FDCs or an FDC cell line, $\mathrm{HK}$, to grow in vitro and to form tumors in vivo. ${ }^{9,10}$ However, L3055 cells become less FDC/HK-dependent after prolonged culture, offering unprecedented opportunities to study transformation in cells of the same origin. We subcloned FDC/HK-dependent and -independent L3055 cells by a limiting dilution method and identified several differentially expressed genes between indolent FDC/HKdependent L3055 clone and aggressive FDC/HK-independent L3055 clone. Among them, CD9 was a marker distin-

Supported by National Institutes of Health grant R01CA121039 to Y.S.C. and Norman Hirshfield Foundation to A.S.F

Accepted for publication March 2, 2010.

Supplemental material for this article can be found on http://ajp. amjpathol.org

Address reprint requests to Dr. Yong Sung Choi, Laboratory of Cellular Immunology, 1514 Jefferson Highway, Ochsner Clinic Foundation, New Orleans, LA 70121. E-mail: ychoi@ochsner.org. 
guishing indolent FDC/HK-dependent B lymphomas from aggressive FDC/HK-independent B lymphomas.

CD9 is a $24-\mathrm{kDa}$ surface membrane protein that belongs to the family of tetraspanin (also called transmembrane 4 superfamily or TM4SF). ${ }^{11}$ It is widely distributed on the surface of normal and malignant cells as well as on a variety of cell lines. ${ }^{11,12}$ It is also constitutively expressed on a subpopulation of B cells. ${ }^{13,14}$ CD9 has been implicated in various biological functions, including cell adhesion, motility, metastasis, growth, signal transduction, differentiation, and sperm-egg fusion. ${ }^{11,15-17}$ The implication of CD9 in cancer has received much attention. An inverse correlation between its expression in primary tumors and the metastatic potential and patient survival rate has been established in various carcinomas. ${ }^{18-21}$ The present report describes a role of CD9 in the progression of human B lymphoma cells.

\section{Materials and Methods}

\section{Reagents}

Mouse anti-human CD9 monoclonal antibodies (mAbs), MM2/57 (unconjugated and phycoerythrin-conjugated) and ALB6 were purchased from Southern Biotech (Birmingham, $A L$ ) and Biovision (Saco, ME), respectively, and two other anti-CD9 mAbs, $1 \mathrm{~B} 6$ and 10E5, were gifts from Dr. J.C. Choe (Department of Microbiology and Immunology, Kangwon National University School of Medicine, Chunchon, Korea). ${ }^{22}$ Fluorescein isothiocyanate-conjugated mouse antibodies against CD82 (53H5) and CD63 (MEM-259) were obtained from eBioscience (San Diego, CA) and Biolegend (San Diego, CA), respectively. Phycoerythrin- or allophycocyanin-conjugated goat anti-mouse IgG were obtained from BD Biosciences (San Diego, CA). 5-Aza-2'-deoxycytidine (5-aza-dc), Trichostatin A (TSA), and carboxyfluorescein diacetate succinimidyl ester (CFSE) were purchased from Sigma (St Louis, MO).

\section{Cell Culture}

L3055, FLK, and HK cells were grown as described previously. ${ }^{10,23,24}$ Wan, BL40, BL41, Mutu, and Chep were a kind gift of Dr. Chris Gregory (Institute of Cell Signaling and School of Biomedical Sciences, University of Nottingham Medical School, Queen's Medical Center, Nottingham, UK). Ramos and Raji cell lines were obtained from ATCC (Manassas, VA). B lymphoma cell lines were maintained in Iscoves modified Dulbecco medium supplemented with $10 \%$ fetal calf serum (FCS), $3 \mathrm{mmol} / \mathrm{L}$ glutamine, $100 \mathrm{U} / \mathrm{ml}$ penicillin $\mathrm{G}$, and $100 \mu \mathrm{g} / \mathrm{ml}$ streptomycin.

\section{DNA Microarray Analysis}

Total RNA was extracted from FDC/HK-dependent L3055-12 and FDC/HK-independent L3055-33 using the RNeasy protect kit (Qiagen, Valencia, CA). RNA samples were prepared from three individual cultures (grown simultaneously) of each cell line. Biotinylated cRNA was hybridized to an Affymetrix chip (HGU133A2.0; Af- fymetrix, Sacramento, CA) according to the manufacturer's instructions by microarray facility of Dana-Farber Cancer Institute. DNA chip analyzer ${ }^{25}$ was used to normalize the Affymetrix gene array data and to obtain perfect match-only model-based expression intensities. An array with a median overall intensity was chosen as the baseline array against which other arrays were normalized at probe intensity level. DNA chip analyzer was used to perform an unsupervised analysis that consisted of gene filtering; that is, excluding genes that lacked sufficient variability across samples and hierarchical clustering of genes and samples (data not shown). To identify the genes whose expression patterns best distinguished indolent FDC/HKdependent L3055-12 from aggressive FDC/HK-independent L3055-33, the permutation distribution of the maximum $t$ statistic was analyzed using the permax test. ${ }^{26}$ To control the overall error rate, the Permax $P$ value was calculated by comparing the observed $t$ statistics for each gene from their log values to the permutation distribution of the largest $t$ statistic obtained over the 54,675 genes contained on the Affymetrix human genome U133 plus 2.0 arrays. For each gene, the $P$ value is the proportion of permutations with the maximum $t$ statistics over all genes greater or equal to the observed value for a particular gene. In these supervised analyses, a test declaring as significant any genes with $P$ of $\leq 0.05$ guarantees that the chance of any false positives being selected is $\leq 5 \%$. Differentially expressed genes (filtered by $P \leq 0.65$ and $\geq 2$-fold increase) from supervised analysis were identified for presentation and further study. A $P$ value $\leq 0.05$ was deemed statistically significant.

\section{Real-Time PCR Analysis}

Total cellular RNA was isolated using RNeasy protect kit (Qiagen). Total RNA was treated with DNase I (Promega, Madison, WI) and reverse transcribed with Promega ImPromll reverse transcriptase kit. Quantitative real-time $\mathrm{PCR}$ reactions were performed in a $10-\mu$ l volume containing 25 ng of cDNA, QuantiTect SYBR Green PCR Master Mix (Qiagen), and $2.5 \mu \mathrm{mol} / \mathrm{L}$ of each gene-specific primer using the DNA Engine Opticon system with PTC200 DNA Engine cycler and CFD-3200 Opticon Detector (MJ Research Inc, Waltham, MA). The following primer sequences were used: DDX3 (forward: 5'-TTCTCAGATGTTTGTTGTGTGGATT-3', reverse: 5'-AAACTTGCTCAAATGCTATTGCTG-3'), ${ }^{27}$ TSLC1 (forward: 5'- GAGTCAACGGATTTGGTCGT-3', reverse: 5'-ATCCACAGTCTTCTGGGTGG-3'), ${ }^{28}$ fibulin 2 (forward: 5'-TCCACCCTAGCTTCCGCTGCCTG-3', reverse: 5'-AAGTCATGGCACGTGGTGCGCTCG-3'), ${ }^{29}$ RGS13 (forward: 5'-ATGAGCAGGCGGAATTGTTGGA-3', reverse: 5'-GAAACTGTTGTTGGACTGCATA-3'), ${ }^{30}$ SDC1 (forward: 5'-GGAGCAGGACTTCACCTTTG-3', reverse: 5'-CTCCCAGCACCTCTTTCCT-3'), ${ }^{31}$ vimentin (forward: 5'-ACCAGGTCCGTGTCCTCGT-3', reverse: 5'-CTGCCCAGGCTGTAGGTG-3'), ANX5 (forward: 5'-TCACCATCTTTGGAACACGA-3', reverse: 5'-TCTCTGCAAGGTAGGCAGGT3'), $\beta 2$ microglobulin (forward: 5'-TGAGTGCTGTCTCCATGTTTGA-3', reverse: 5'-TCTGCTCCCCACCTCTAAGTTG-3'). Primers for CBS, ANX2, CD9, SNRPN, and $\angle T \beta$ 
A

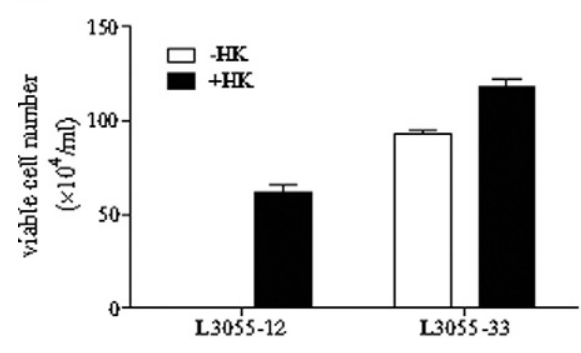

B

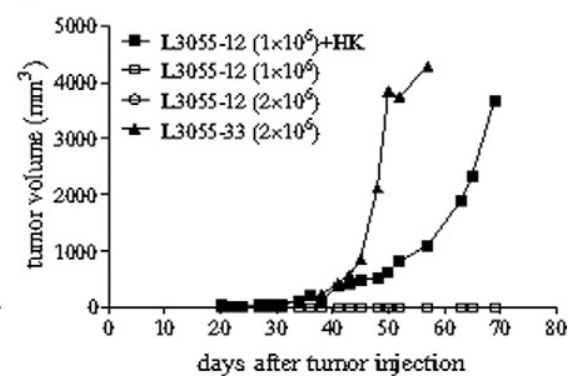

Figure 1. L3055-33 does not require FDC/HK cells for in vitro cell growth and in vivo tumor formation, but L3055-12 does. A: L3055-33 and L3055-12 $\left(2 \times 10^{4}\right.$ cells per well, 24 -well plate were cultured with or without FDC/HK cells $\left(2 \times 10^{4}\right.$ cells per well, irradiated 5000Rad) in RPMI media containing 10\% FCS for four days, and viable cells were counted. B: Tumor growth curves of a typical experiment. SCID/NOD mice were subcutaneously injected with indicated numbers of L3055-33 or L3055-12 alone or in combination with FDC/HK cells. Tumor volume was measured twice a week by a caliper for 70 days. were purchased from Qiagen. After cycling, the specificity of amplification was validated by generating a melting curve using slow denaturation of the PCR products. $\mathrm{Ct}$ values were analyzed with the $\Delta \Delta \mathrm{Ct}$ method using $\beta 2$ microglobulin expression for normalization.

\section{Demethylation and Histone Deacetylase Inhibition}

CD9-negative L3055-33 and Wan cells were seeded at a density of $6 \times 10^{5} / \mathrm{ml}$ and incubated with TSA or 5-aza-dc for 24 or 48 hours, respectively.

\section{Flow Cytometry}

B lymphoma cells were incubated with mAbs for 15 minutes at $4^{\circ} \mathrm{C}$. Unconjugated $\mathrm{mAbs}$ were detected by phycoerythrin- or allophycocyanin-labeled goat anti-mouse Ig. After washing with PBS containing $0.2 \%$ BSA and $0.1 \%$ sodium azide, cells were fixed with $1 \%$ paraformaldehyde and analyzed by flow cytometry.

\section{In Vitro Lymphoma Cell Proliferation Assay}

B lymphoma cells $\left(2 \times 10^{4}\right.$ cells per well, if not otherwise indicated) were cultured in 24-well plates in the absence or presence of irradiated $\mathrm{HK}$ cells (5000 rad, $2 \times 10^{4}$ cells per well). At the time of harvest, B lymphoma cells were collected, and viable cells were enumerated by counting the cells with intact morphology after staining with trypan blue. Contaminating HK cells were excluded in cell counting by their larger cell size.

For cell division experiments, B lymphoma cells were labeled with $1 \mu \mathrm{mol} / \mathrm{L} \mathrm{CFSE}$ at $37^{\circ} \mathrm{C}$ for 10 minutes. FCS was added to stop staining, and then labeled cells were washed with culture medium. At the end of the culture, the CFSE intensity was measured by FACSCalibur.

\section{In Vivo Lymphomagenesis}

Female 8-week-old SCID/NOD mice (Jackson Laboratory, Bar Harbor, ME) were subcutaneously injected with lymphoma cells for tumor formation. For this purpose, L3055-12 and L3055-33 cells grown in a log phase were collected from the in vitro culture and injected with or without HK cells into mice subcutaneously. All cell suspensions (0.1 ml/injection site) were injected with a 25gauge needle in the posterior flank of SCID/NOD mice.
Tumors were measured in three dimensions twice a week with a caliper for 70 days. Tumor size was expressed in volume (volume $=$ length $\times$ width $\times$ height, in $\mathrm{mm}^{3}$ ).

\section{Results \\ Clonal Selection of FDC/HK-Independent L3055 Cells}

We previously showed that a lymphoma cell line of the GC origin, L3055, requires FDCs or an FDC cell line, HK, to grow in vitro and to form tumors in vivo. ${ }^{9,10}$ In the absence of FDC/HK cells, these cells undergo apoptosis and do not form tumors. L3055 cells, however, become less FDC/HK-dependent after prolonged culture. To obtain an FDC/HK-independent L3055 clone, we performed a limiting dilution in the presence or absence of FDC/HK cells. After a 2-week culture, the surviving cells from each well were collected and assayed for FDC/HK dependency. We selected the most FDC/HK-dependent clone (L3055-12) and FDC/HK-independent clone (L3055-33) among these clones. As shown in Figure 1A, L3055-33 survived and proliferated in the absence of FDC/HK cells in contrast to L3055-12. Furthermore, L3055-33 formed tumors without FDC/HK cells in SCID/NOD mice (Figure 1B). In contrast, no tumor formation was observed when the same number of L3055-12 was injected alone without FDC/HK cells (Figure 1B). This result suggests that L3055-33 have adapted detour growth pathways, allowing the cells to bypass FDC/HK requirement for their growth.

\section{Gene Expression Profiling of FDC/HK-Dependent L3055-12 and-Independent L3055-33 Clones}

Global gene expression profiles were obtained and the microarray data analyzed using supervised learning to identify the genes that distinguish indolent FDC/HK-dependent L3055-12 from aggressive FDC/HK-independent L3055-33. As listed in Tables 1 and 2, expression levels of 18 genes were at least twofold higher in L3055-12 compared with L3055-33 ( $P \leq 0.65$ ), whereas expression of 7 genes was at least twofold higher in L3055-33 compared with L3055-12 ( $P \leq 0.65)$. There was one statistically significant $(P \leq 0.05)$ difference in gene expression between L3055-12 and L3055-33; CD9 was expressed at a 53-fold increase in L3055-12 compared 
Table 1. Upregulated Genes in FDC/HK-Dependent L3055-12 Clone

\begin{tabular}{clc}
\hline Affymetrix probe no. & \multicolumn{1}{c}{ Description } & Signal fold ratio (L3055-12/L3055-33) P value \\
\hline 201005_at & CD9 antigen (p24) & 52.952685 \\
221606_s_at & Nucleosomal binding protein 1 & 13.419818 \\
203386_at & TBC1 domain family, member 4 & 13.143097 \\
201426_s_at & Vimentin & 11.436873 \\
225710_at & Homo sapiens cDNA FLJ34013 fis, clone FCBBF2002111 & 11.125569 \\
201286_at & Syndecan 1 (SDC1) & 10.740027 \\
203886_s_at & Fibulin 2 & 9.444904 \\
209031_at & Immunoglobulin superfamily, member 4 (TSLC1) & 8.532908 \\
220643_s_at & Fas apoptotic inhibitory molecule & 6.181087 \\
201210_at & DEAD (Asp-Glu-Ala-Asp) box polypeptide 3, X-linked (DDX3) & 5.838330 \\
1561562_at & Homo sapiens cDNA clone IMAGE:5297041, partial cds & 4.830113 \\
203068_at & KIAA0469 gene product & 3.253889 \\
207339_S_at & Lymphotoxin beta (TNF superfamily, member 3) (LT $\beta)$ & 3.087912 \\
237646_x_at & KIAA0720 protein & 3.041645 \\
243561_at & Homo sapiens transcribed sequences & 2.879987 \\
230970_at & Homo sapiens transcribed sequences & 2.630661 \\
243149_at & Homo sapiens transcribed sequences & 2.381888 \\
238875_at & Homo sapiens transcribed sequences & 2.298306 \\
\hline
\end{tabular}

with L3055-33 ( $P=0.05)$. To validate the microarray data, the expression level of 12 selected genes, including 7 genes up-regulated in L3055-12 (CD9, fibulin2, LT $\beta$, DDX3, SDC1, vimentin, and TSLC1) and 5 genes upregulated in L3055-33 (SNRPN, RGS13, ANX2, CBS, and ANX5), were compared by real-time PCR between L3055-12 and L3055-33. The results of one representative experiment for each gene are shown in Figure 2. The expression of all 12 genes tested was consistent with the expression patterns observed in the microarray, showing an increased expression of CD9, Fibulin 2, $L T \beta, D D X 3$, SDC1, vimentin, and TSLC1 in L3055-12 (Figure 2A) and an increased expression of SNRPN, RGS13, ANX2, CBS, and ANX5 in L3055-33 (Figure 2B).

\section{CD9-Positive Cells Grow Slower and Are More Dependent on FDC/HK Cells Compared with CD9-Negative Cells}

Because CD9 has been reported to correlate with tumor progression, we decided to focus on the CD9 gene for further investigation. CD9 exhibited a dramatic difference between FDC/HK-dependent L3055-12 and -independent L3055-33 (more than 800-fold increase in L3055-12 by real-time PCR). This difference at the mRNA level was confirmed at the protein level by FACS analysis (Figure $3 A)$. In contrast, two other tetraspanin proteins, CD82 and CD63, that are also reported to correlate with tumor pro- gression $^{32-38}$ did not have any difference in expression between L3055-12 and L3055-33 (Figure 3A).

We further examined CD9 expression on other mature B lymphoma cell lines (Table 3). Overall, most of the cell lines did not express CD9 (Raji, BL41, Mutu, Ramos, Chep, and FLK), but two cell lines expressed CD9 (Wan and BL40). Wan and BL40 exhibited high but quite homogeneous expression of CD9 similar to L3055-12. Interestingly, Wan and BL40 progressively lost CD9 expression after the prolonged in vitro culture similar to the L3055 cell line (Figure 3B).

To examine the relationship between CD9 expression and cell growth, we separated CD9-positive and CD9negative populations from L3055, Wan, and BL40 cell lines using a MACS column (Figure 3B) and compared their cell growth. After 3 to 4 days of culture, the recovery of CD9-negative population was higher than CD9-positive population (Figure 3C). The difference in cell growth was more dramatic when cells were cultured in harsh conditions (e.g., smaller seeding numbers). Because CD9 expressing L3055-12 cells but not L3055-33 were dependent on FDC/HK cells for their growth (Figure 1A), we also examined the growth of CD9-positive and -negative populations from Wan and BL40 in the presence of FDC/HK cells. Consistent with observation made with the L3055-12 and -33 cells, CD9-positive population was relatively dependent on FDC/HK cells for growth compared with the CD9negative population (Figure 3D). The differences in

Table 2. Upregulated Genes in FDC/HK-Independent L3055-33 Clone

\begin{tabular}{llrr}
\hline $\begin{array}{c}\text { Affymetrix } \\
\text { probe no. }\end{array}$ & \multicolumn{1}{c}{ Description } & Signal fold ratio (L3055-33/L3055-12) & $P$ value \\
\hline 242742_at & $\begin{array}{c}\text { Homo sapiens transcribed sequence with moderate } \\
\text { similarity to protein ref }\end{array}$ & 24.4961030 & \\
& Small nuclear ribonucleoprotein polypeptide N (SNRPN) & 20.6911160 & 6.10 \\
206042_x_at & Cystathionine-beta-synthase (CBS) & 6.9362115 & 0.10 \\
1553972_a_at & Annexin A2 (ANX2) & 5.7030442 & 0.58 \\
210427_x_at & Regulator of G-protein signalling 13 (RGS13) & 3.7282627 & 0.30 \\
210258_at & Annexin A5 (ANX5) & 2.8125177 & 0.60 \\
200782_at & Small nuclear ribonucleoprotein polypeptide N & & 0.35 \\
201522_x_at & S & & \\
\hline
\end{tabular}


A

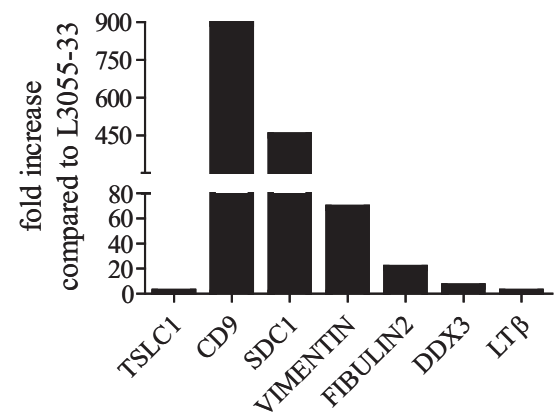

upregulated genes in L3055-12
B

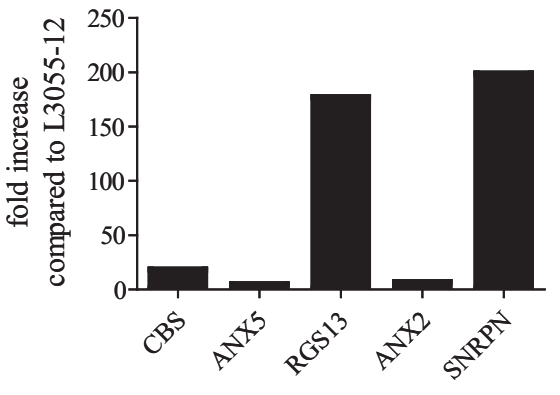

Figure 2. Validation of microarray data in L3055-12 and L3055-33 by quantitative real-time PCR. The relative fold increase of each transcript in L3055-12 (A) and L3055-33 (B) is shown in comparison with the level of the transcript in L3055-33 and L3055-12, respectively.
FDC/HK dependency between CD9-positive and CD9negative population for their growth were more dramatic when the initially seeded cell numbers were low.

The slower growth of CD9-positive population is an intrinsic characteristic but not attributed to a cytotoxic effect of anti-CD9 antibody bound to B lymphoma cells during separation procedure because the addition of anti-CD9 to the cultures of CD9-positive cell lines (L3055-12, Wan, and BL40) did not have any influence on viable cell recovery (Supplemental Figure S1 at http://ajp.amjpathol.org). In addition, similar results were observed with different anti-CD9 antibodies (clones ALB6, 1B6, and 10E5; data not shown).

\section{CD9-Positive Population Is More Susceptible to Apoptosis than CD9-Negative Population}

Because slower cell growth can result either from an increase in the rate of apoptosis or from a slower pro-
A

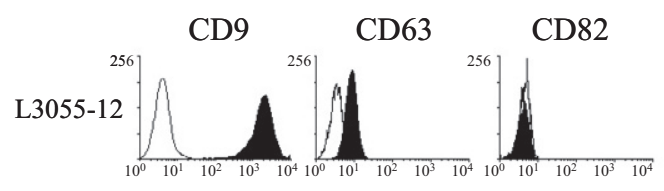

\section{B}

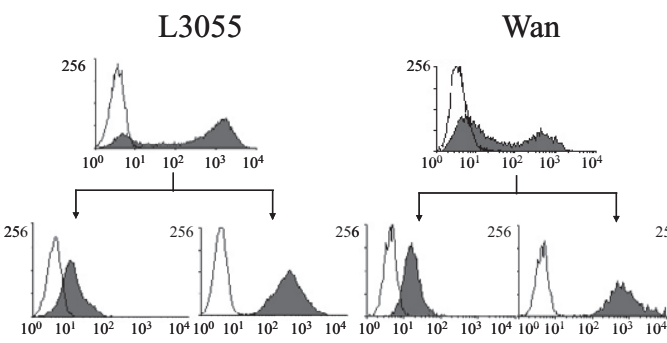

Wan

BL40

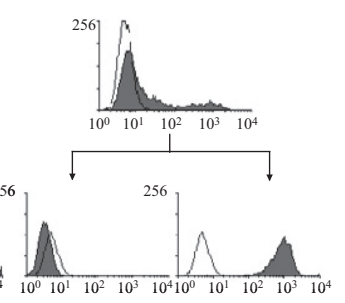

BL40
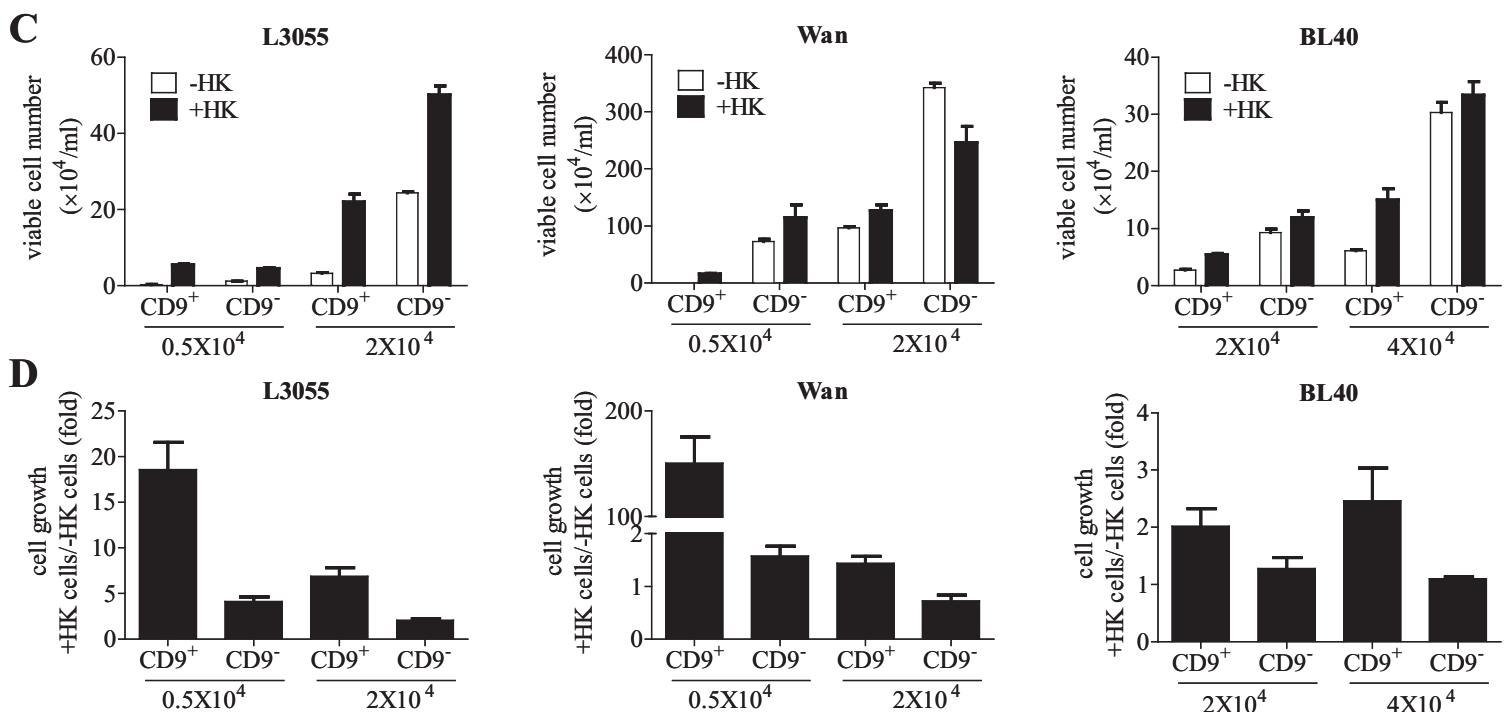

Figure 3. CD9-negative population grows faster but less dependent on FDC/HK cells compared with the CD9-positive population. Expression levels of CD9, CD63, and CD82 in L3055-12 and L3055-33 were analyzed by flow cytometry. Open histograms represent isotype control, and shaded histograms represent the surface CD9, CD63, and CD82 expression (A). CD9-positive and CD9-negative populations were separated using a MACS column from L3055, Wan, and BL40 (B) and then cultured in the presence or absence of FDC/HK cells as indicated (C and $\mathbf{D}$ ). At the end of the culture, cells were harvested and viable cell numbers were counted $(\mathbf{C})$ and FDC/HK cell dependency was determined by dividing viable cell numbers in the presence of FDC/HK cells by viable cell numbers in the absence FDC/HK cells (D). 
Table 3. Expression of Tetraspanins in B Lymphoma Cell Lines*t

\begin{tabular}{cccccccccccc}
\hline Tetraspanin & L3055-12 & L3055-33 & Wan $^{\ddagger}$ & BL-40 & BL-41 & FLK1 & Raji & Mutu & Chep & Ramos \\
\hline CD9 & +++ & - & $+++(-)$ & +++ & - & - & - & - & - & - \\
CD63 & + & + & $++(++)$ & - & ++ & + & + & + & ++ & + \\
CD82 & - & - & $-(-)$ & - & - & - & ++ & + & + & - \\
\hline
\end{tabular}

*The measured parameter was the mean fluorescence intensity $(\mathrm{MFI})$. Expression quantification is presented from the lowest $(0<\mathrm{MFI}<10[+])$ to the highest $(100<\mathrm{MFI}[+++])$ levels.

†For CD9 expression, two mAbs (MM2/57 and 10E5) gave similar results.

${ }^{\ddagger}$ Indicates CD9 ${ }^{-}$clone.

gression through the cell cycle, we examined these two possibilities. Wan and BL40 cells that contain both CD9positive and -negative populations were labeled with CFSE and cultured in the presence or absence of FDC/HK cells for 3 days. The cells were collected every day and analyzed for the percentage of CD9-positive and -negative populations and their CFSE profiles. As shown in Figure 4, the percentage of CD9-positive population decreased over time. The decrease in the percentage of CD9-positive population was more dramatic when B lymphoma cells were cultured in the absence of FDC/HK cells compared to those with FDC/HK cells. However, there was no difference between CD9-positive and -negative populations in CFSE profiles during the culture period. The data suggest that slower growth of CD9-positive population is attributed to susceptibility to apoptosis rather slower cell cycle progression and confirm that FDC/HK cells prevent apoptosis of B lymphoma cells as shown previously. ${ }^{10}$

\section{CD9 Expression Is Epigenetically Regulated}

To understand the mechanisms implicated in the loss of CD9 protein expression in B lymphomas, we examined whether modification of histones and/or DNA methylation are involved in the down-regulation of CD9 expression because both mechanisms are proven to be important regulators of gene expression. To this end, we cloned CD9-negative cells from Wan cell line heterogeneous in CD9 expression levels and treated them with Histone deacetylase inhibitor TSA and demethylation agent 5-aza-dc along with L3055-33 cells. Treatment with 40 nmol/L TSA for 24 hours induced a 33-fold increase in CD9 mRNA levels in the L3055-33 as determined by quantitative real-time PCR (Figure 5A). In contrast, 5 $\mu \mathrm{mol} / \mathrm{L} 5$-aza-dc up to 2 days resulted only in a modest 4.8-fold increase in CD9 mRNA levels. Either higher concentration of 5-aza-dc (up to $10 \mu \mathrm{mol} / \mathrm{L}$ ) or longer incubation (up to 4 days) did not increase CD9 expression
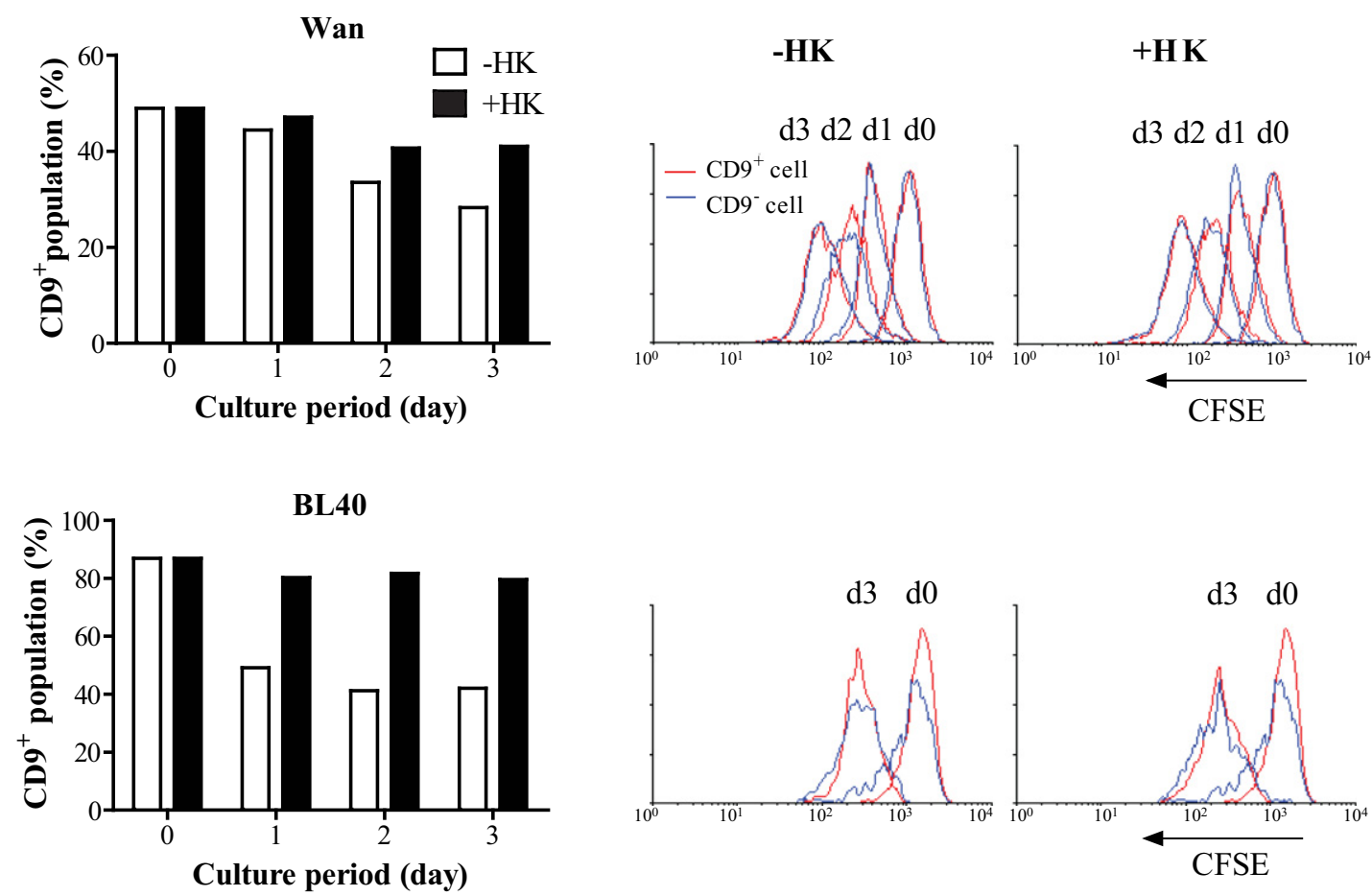

Figure 4. CD9-positive population is more susceptible to apoptosis. Heterogeneous Wan and BL40 cells in CD9 expression were labeled with CFSE and cultured in the presence or absence of FDC/HK cells in IMDM media without FCS for three days. Cells were collected at the indicated time points, and then CFSE profile and the percentage of CD9-positive population were determined by FACS staining. 
A

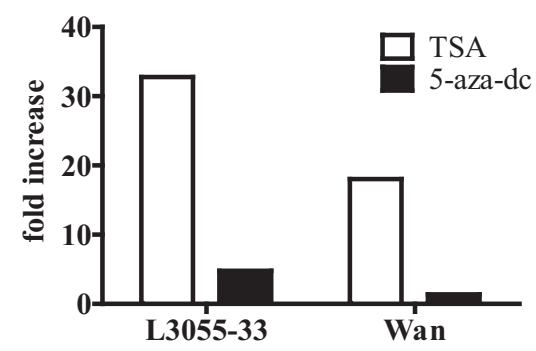

B Medium TSA 5-aza-dc

L3055-33
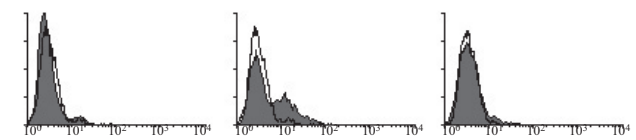

Wan
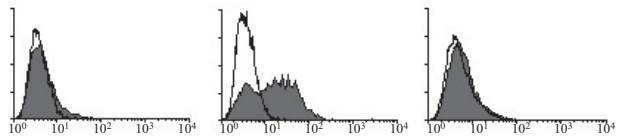

Figure 5. CD9 expression is epigenetically regulated in B lymphoma cells. CD9-negative L3055-33 and Wan were treated with TSA (40 and $50 \mathrm{nmol} / \mathrm{L}$, respectively) and $5 \mu \mathrm{mol} / \mathrm{L} 5$-aza-dc. A: Quantitative real-time PCR analysis showing relative fold induction of CD9 mRNA after drug treatment compared with control. B: Flow cytometric analysis showing that induced membrane CD9 expression after drug treatment compared with control. Open histograms represent isotype control, and shaded histograms represent CD9 specific staining.

further. Similar data were obtained with CD9-negative Wan clone with lower fold changes of CD9 mRNA after treatment. CD9 protein expression using flow cytometric analysis was consistent with the changes observed in CD9 mRNA levels (Figure 5B). These data indicate that deacetylation is the key epigenetic mechanism in regulating CD9 expression in B lymphomas.

\section{Assessment of CD9 Expression in Clinical Samples}

To further confirm the down-regulation of CD9 expression during disease progression in B lymphomas, we compared CD9 expression in microarray dataset of matched pairs of follicular lymphoma (FL) and their transformed counterparts, diffuse large B cell lymphoma (DLBCL) derived from the same individual ${ }^{39}$ because transformation of FL to more aggressive DLBCL generally takes rapidly progressive clinical course often refractory to treatment and associated with short survival. ${ }^{40}$

Six of 14 cases (IL105, IL111, IL119, IL120, IL122, and IL125) showed a decrease in CD9 expression (Figure 6). Among these cases, three cases exhibited at least threefold decrease (IL111, IL119, and IL120). In contrast, three cases showed a moderate increase (less than threefold; IL117, IL121, and IL126) after FL higher-grade transformation. Five cases (IL112, IL114, IL116, IL123, and IL124) showed no changes in CD9 expression. However, in most of these cases CD9 expression was low in the FL samples, suggesting that CD9 was down-regulated before transformation to DLBCL in these cases. Because relatively high number of cases (five of 14 cases) in this analysis expressed low levels of CD9 be-

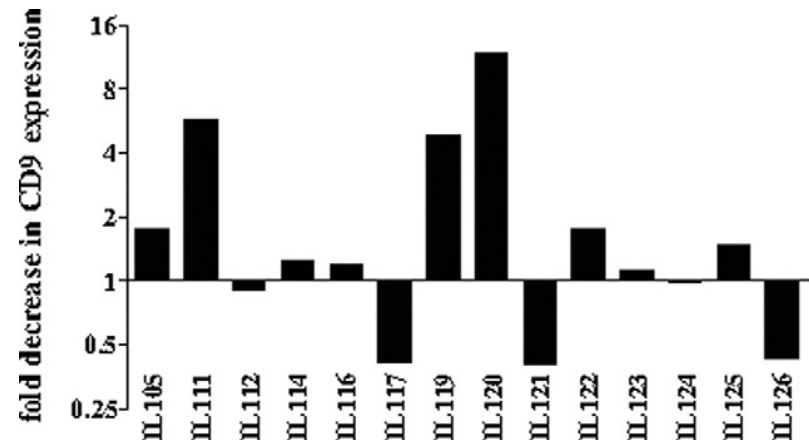

Figure 6. Comparison of CD9 expression in paired follicular lymphomas and its transformed counterpart diffuse large B cell lymphomas. CD9 expression during transformation was compared using microarray dataset of matched pairs of FL and DLBCL from the same patient. Fold decrease in CD9 expression was calculated from CD9 expression levels in DLBCL divided by those of FL.

fore transformation, definitive conclusions about relation between CD9 expression and tumor progression require further study in larger groups of patients.

\section{Discussion}

Histological transformation is a pivotal event in the natural history of cancers including lymphomas, typically with more aggressive clinical behavior. We previously showed that a lymphoma cell line of the GC origin, L3055, requires the stromal cells in the GC, FDC, to grow in vitro and to form tumors in vivo., 9,10 L3055 cells, however, became less FDC/HK-dependent after prolonged culture. Here, we establish a FDC/HK-independent L3055 clone; i) It survives in the absence of HK cells in in vitro culture, and ii) it forms tumors without HK cells when inoculated into SCID/NOD mice. This transition from FDC/ HK-dependent lymphoma to FDC/HK-independent lymphoma is analogous to the transformation of indolent $\mathrm{FL}$ to more aggressive DLBCL, which occurs in a substantial number of patients with FL, with a risk of $3 \%$ per year. ${ }^{41}$ In this process a more virulent subclone of cells emerges, typically associated with the loss of follicular histological architecture, a rapidly progressive clinical course refractory to treatment, and short survival. ${ }^{42}$ Thus, our FDC/HK-dependent and FDC/HK-independent L3055 subclones (L3055-12 and L3055-33) from the same parent cell line offer unprecedented opportunities to study the genetic changes associated with the transformation process, rather than random differences associated with comparison to different B lymphoma cell lines.

To identify differentially expressed genes between FDC/HK-dependent and FDC/HK-independent L3055 cells, DNA microarray was performed and data were analyzed. CD9 was selected for further study because it was a gene with greatest differences in expression. In addition, CD9 expression has been reported to correlate with tumor progression in various tumors but has not been studied in B lymphomas. ${ }^{43-48}$ CD9 is highly expressed on less aggressive L3055-12 but not on more aggressive L3055-33. The reduced or loss of CD9 ex- 
pression upon transition of less aggressive $B$ lymphomas to advanced B lymphomas appeared not to be limited to L3055 cells because CD9 expressing two other B lymphoma cell lines (i.e., Wan and BL40) also gradually lost CD9 after prolonged in vitro culture. Furthermore, the resulting CD9-negative population from these cell lines grew faster than CD9-positive population. The greater capacity of CD9-negative population in growth appears to be attributed to its better survival rather than faster cell proliferation. However, it is not certain whether CD9 expression per se negatively affects the B lymphoma cell growth. The effect of CD9 in cell growth has shown to be various depending on cell types. Ectopic expression of CD9 in lung cancer cell line did not change tumor growth, ${ }^{49}$ but that in colon and prostate cancer cell lines negatively affected the cell growth. ${ }^{50,51}$ It is possible that CD9-negative population may acquire some advantageous characteristics for growth (eg, loss of proapoptotic genes or gain of antiapoptotic genes).

Although we clearly show that CD9 is a surface marker distinguishing the cells derived from the same parent but with different capacity of growth, it is also possible that CD9 has a functional role in B lymphoma cells. CD9positive population was more dependent on FDC/HK cells than CD9-negative population for their growth, suggesting that CD9 may play a role in physical contacts between $\mathrm{B}$ lymphomas and stromal cells (FDC/HK) which may facilitate delivery of proliferative signals from stromal cells. A similar observation has been made in multiple myeloma (MM). CD9 is expressed in stroma-dependent MM clones but not in stroma-independent clones. ${ }^{52,53}$ In addition, GC-B cells that require interactions with FDCs for their survival express high levels of CD9 (supplemental Figure S2 at http://ajp.amjpathol.org). Indeed, CD9 has been reported to be involved in heterotypic cellcell interactions. ${ }^{22,54-56}$ Furthermore, CD9 has been shown to increase biological activity of growth factor such as HB-EGF. ${ }^{57,58}$ Thus, CD9 may contribute to cell growth by collaborating with survival or growth factors produced by FDC/HK cells. The heterotypic interaction between $B$ lymphoma cells and FDC/HK cells through CD9 molecule may be important in FDC/HK dependent, indolent stage; however, the cells with reduced CD9 expression in $\mathrm{FDC} / \mathrm{HK}$-independent advanced stage may bypass this mechanism. Therefore, it will be of interest to investigate whether CD9 plays roles in interaction between FDC/HK and B lymphoma cells.

Our experimental data are in line with clinical results that CD9 expression inversely correlated to tumor progression in various tumors. Along the line, CD9 was also suggested as a prognostic marker in some cancers because patients with reduced CD9 expression showed a less favorable prognosis than those with positive expression. ${ }^{18,19,21,35,59}$ Regarding B cell malignancies, CD9 expression was reported to be inversely associated with disease-free survival in diffuse $B$ lymphoma patients and inversely correlated with disease activity and survival of MM patients. ${ }^{48,59}$ Along with CD9, other tetraspanin members CD82 and CD63 have been reported to be inversely related with tumor progression in various tumors. ${ }^{32-38}$ However, we could not observe any correla- tion between tumor progression and these tetraspanins in B lymphoma cell lines examined (Table 3).

CD9 silencing observed during B lymphoma progression appears to be epigenetically regulated. Consistent with the notion that histone modifications are more dynamic and occur earlier in epigenetic silencing while DNA methylation maintains long-term silencing of genes that had already been switched off by histone modifications, we found that histone deacetylation is the key epigenetic mechanism in regulating CD9 expression in B lymphomas. The similar observations were made in lung cancer and MMs. ${ }^{48,60}$ Although it has recently been reported that mRNA modifications (i.e., deletion, insertion, or mutations) could play a role in regulating CD9 during prostate cancer progression, ${ }^{47}$ we could not detect any deletion and insertion in CD9 mRNA when full length of coding region of CD9 was amplified. However, because the possibility precludes CD9 detection depending on epitopes recognized by CD9 antibody used for immunohistochemical staining, it should be taken into consideration.

To correlate CD9 expression and B lymphoma progression in clinical setting, we compared CD9 expression of matched pairs of $\mathrm{FL}$ and their transformed counterparts, DLBCL occurring within the same individual (14 cases) from microarray. ${ }^{39}$ While six of 14 cases showed a decrease in CD9 expression during FL higher-grade transformation, three cases showed a moderate increase in CD9 expression. No changes were observed in five cases, in most of which CD9 expression was low in $\mathrm{FL}$ even before transformation to DLBCL. Overall CD9 downregulation was not uniform in all cases examined. However, because relatively limited number of cases was included for this analysis and CD9 was expressed at very low levels even before transformation in high percentage of the cases (five of 14 cases), definitive conclusions about relation between CD9 expression and tumor progression require further study in larger groups of patients. Nevertheless, it should be noted that normal counterpart of B lymphomas, GC-B cells, express high levels of CD9 (supplemental Figure S2 at http://ajp.amjpathol.org) while most of B lymphoma cell lines and B lymphomas from patients express low levels or do not express CD9. ${ }^{14}$

In conclusion, loss of CD9 expression in B lymphomas may be a useful marker for FDC dependency or tumor progression. Further studies in primary tumors are needed to establish CD9 role in the process of higher grade transformation of lymphoma.

\section{References}

1. Stevenson F, Sahota S, Zhu D, Ottensmeier C, Oscier D, Hamblin T: Insight into the origin and clonal history of B-cell tumors as revealed by analysis of immunoglobulin variable region genes. Immunol Rev 1998, 162:247-259

2. Kuppers R, Klein U, Hansmann ML, Rajewsky K: Cellular origin of human B-cell lymphomas. N Engl J Med 1999, 341:1520-1529

3. Pascual V, Liu Y-J, Magalski A, de Bouteiller O, Banchereau J, Capra JD: Analysis of somatic mutation in five $B$ cell subsets of human tonsil. J Exp Med 1994, 180:329-339

4. Kimoto H, Nagaoka H, Adachi $Y$, Mizuochi T, Azuma T, Yagi T, Sata T, Yonchara S, Tsunetsugu-Yokota Y, Taniguchi M, Takemori T: Ac- 
cumulation of somatic hypermutation and antigen-driven selection in rapidly cycling surface $\mathrm{Ig}^{+}$germinal center (GC) B cells which occupy GC at a high frequency during the primary anti-hapten response in mice. Eur J Immunol 1997, 27:268-279

5. Kosco-Vilbois MH, Scheidegger D: Follicular dendritic cells: antigen retention. B cell activation, and cytokine production. Curr Top Microbiol Immunol 1995, 201:69-82

6. Choi YS: Differentiation and apoptosis of human germinal center B-lymphocytes. Immunol Res 1997, 16:161-174

7. Petrasch S, Kosco M, Perez-Alvarez C, Schmitz J, Brittinger G: Proliferation of non-Hodgkin-lymphocytes in vitro is dependent upon follicular dendritic cell interactions. Br J Haema 1992, 80:21-26

8. Shiozawa E, Yamochi-Onizuka T, Yamochi T, Yamamoto Y, Naitoh H, Kawakami K, Nakamaki T, Tomoyasu S, Kushima M, Ota H: Disappearance of CD21-positive follicular dendritic cells preceding the transformation of follicular lymphoma: immunohistological study of the transformation using CD21, p53, Ki-67, and P-glycoprotein. Pathol Res Pract 2003, 199:293-302

9. Choe J, Li L, Zhang X, Gregory CD, Choi YS: Distinct role of follicular dendritic cells and $T$ cells in the proliferation, differentiation, and apoptosis of a centroblast cell line. L3055. J Immunol 2000, 164:56-63

10. Li L, Yoon SO, Fu DD, Zhang X, Choi YS: Novel follicular dendritic cell molecule, 8D6, collaborates with CD44 in supporting lymphomagenesis by a Burkitt lymphoma cell line, L3055. Blood 2004, 104:815-821

11. Maecker HT, Todd SC, Levy S: The tetraspanin superfamily: molecular facilitators. FASEB J 1997, 11:428-442

12. Sincock PM, Mayrhofer G, Ashman LK: Localization of the transmembrane 4 superfamily (TM4SF) member PETA-3 (CD151) in normal human tissues: comparison with $\mathrm{CD} 9, \mathrm{CD} 63$, and alpha5beta1 integrin. J Histochem Cytochem 1997, 45:515-525

13. Won WJ, Kearney JF: CD9 is a unique marker for marginal zone B cells, B1 cells, and plasma cells in mice. J Immunol 2002, 168:5605-5611

14. Barrena S, Almeida J, Yunta M, Lopez A, Fernandez-Mosteirin N, Giralt M, Romero M, Perdiguer L, Delgado M, Orfao A, Lazo PA: Aberrant expression of tetraspanin molecules in B-cell chronic lymphoproliferative disorders and its correlation with normal B-cell maturation. Leukemia 2005, 19:1376-1383

15. Boucheix C, Rubinstein E: Tetraspanins. Cell Mol Life Sci 2001, 58:1189-1205

16. Hemler ME: Tetraspanin functions and associated microdomains. Nat Rev Mol Cell Biol 2005, 6:801-811

17. Zoller M: Tetraspanins: push and pull in suppressing and promoting metastasis. Nat Rev Cancer 2009, 9:40-55

18. Miyake M, Nakano K, Itoi SI, Koh T, Taki T: Motility-related protein-1 (MRP-1/CD9) reduction as a factor of poor prognosis in breast cancer. Cancer Res 1996, 56:1244-1249

19. Higashiyama M, Doi O, Kodama K, Yokouchi H, Adachi M, Huang CL, Taki T, Kasugai T, Ishiguro S, Nakamori S, Miyake M: Immunohistochemically detected expression of motility-related protein-1 (MRP-1/ CD9) in lung adenocarcinoma and its relation to prognosis. Int $J$ Cancer 1997, 74:205-211

20. Huang Cl, Kohno N, Ogawa E, Adachi M, Taki T, Miyake M: Correlation of reduction in MRP-1/CD9 and KAl1/CD82 expression with recurrences in breast cancer patients. Am J Pathol 1998, 153:973-983

21. Mori M, Mimori K, Shiraishi T, Haraguchi M, Ueo H, Barnard GF, Akiyoshi T: Motility related protein 1 (MRP1/CD9) expression in colon cancer. Clin Cancer Res 1998, 4:1507-1510

22. Ko EM, Lee IY, Cheon IS, Kim J, Choi JS, Hwang JY, Cho JS, Lee DH, Kang D, Kim SH, Choe J: Monoclonal antibody to CD9 inhibits platelet-induced human endothelial cell proliferation. Mol Cells 2006, 22:70-77

23. Kim H-S, Zhang X, Choi YS: Activation and proliferation of follicular dendritic cell-like cells by activated T Iymphocytes. J Immunol 1994, 153:2951-2961

24. Kagami Y, Jung J, Choi YS, Osumi K, Nakamura S, Morishima Y, Seto M: Establishment of a follicular lymphoma cell line (FLK-1) dependent on follicular dendritic cell-like cell line HK. Leukemia 2001, 15:148-156

25. Li C, Wong WH: Model-based analysis of oligonucleotide arrays: expression index computation and outlier detection. Proc Natl Acad Sci USA: 2001, 98:31-36
26. Mutter GL, Baak JP, Fitzgerald JT, Gray R, Neuberg D, Kust GA, Gentleman R, Gullans SR, Wei LJ, Wilcox M: Global expression changes of constitutive and hormonally regulated genes during endometrial neoplastic transformation. Gynecol Oncol 2001, 83:177-185

27. Chao $\mathrm{CH}$, Chen CM, Cheng PL, Shih JW, Tsou AP, Lee YH: DDX3, a DEAD box RNA helicase with tumor growth-suppressive property and transcriptional regulation activity of the p21waf1/cip1 promoter, is a candidate tumor suppressor. Cancer Res 2006, 66:6579-6588

28. Lung HL, Cheung AK, Xie D, Cheng Y, Kwong FM, Murakami Y, Guan XY, Sham JS, Chua D, Protopopov AI, Zabarovsky ER, Tsao SW, Stanbridge EJ, Lung ML: TSLC1 is a tumor suppressor gene associated with metastasis in nasopharyngeal carcinoma. Cancer Res 2006, 66:9385-9392

29. Liton PB, Liu X, Stamer WD, Challa P, Epstein DL, Gonzalez P: Specific targeting of gene expression to a subset of human trabecular meshwork cells using the chitinase 3-like 1 promoter. Invest Ophthalmol Vis Sci 2005, 46:183-190

30. Shi GX, Harrison K, Wilson GL, Moratz C, Kehrl JH: RGS13 regulates germinal center $\mathrm{B}$ lymphocytes responsiveness to CXC chemokine ligand (CXCL)12 and CXCL13. J Immunol 2002, 169:2507-2515

31. Sun $\mathrm{H}$, Berquin IM, Edwards IJ: Omega-3 polyunsaturated fatty acids regulate syndecan-1 expression in human breast cancer cells. Cancer Res 2005, 65:4442-4447

32. Atkinson B, Ernst CS, Ghrist BF, Herlyn M, Blaszczyk M, Ross AH, Herlyn D, Steplewski Z, Koprowski H: Identification of melanomaassociated antigens using fixed tissue screening of antibodies. Cancer Res 1984, 44:2577-2581

33. Atkinson B, Ernst CS, Ghrist BF, Ross AH, Clark WH, Herlyn M, Herlyn D, Maul G, Steplewski Z, Koprowski H: Monoclonal antibody to a highly glycosylated protein reacts in fixed tissue with melanoma and other tumors. Hybridoma 1985, 4:243-255

34. Kondoh M, Ueda M, Ichihashi M, Mishima Y: Decreased expression of human melanoma-associated antigen ME491 along the progression of melanoma pre-canceroses to invasive and metastatic melanomas. Melanoma Res 1993, 3:241-245

35. Adachi M, Taki T, leki $Y$, Huang CL, Higashiyama M, Miyake M: Correlation of KAl1/CD82 gene expression with good prognosis in patients with non-small cell lung cancer. Cancer Res 1996, 56:1751-1755

36. Guo X, Friess H, Graber HU, Kashiwagi M, Zimmermann A, Korc M, Buchler MW: KAl1 expression is up-regulated in early pancreatic cancer and decreased in the presence of metastases. Cancer Res 1996, 56:4876-4880

37. Lombardi DP, Geradts J, Foley JF, Chiao C, Lamb PW, Barrett JC: Loss of KAl1 expression in the progression of colorectal cancer. Cancer Res 1999, 59:5724-5731

38. Yang X, Wei L, Tang C, Slack R, Montgomery E, Lippman M: KAl1 protein is down-regulated during the progression of human breast cancer. Clin Cancer Res 2000, 6:3424-3429

39. Lossos IS, Alizadeh AA, Diehn M, Warnke R, Thorstenson Y, Oefner PJ, Brown PO, Botstein D, Levy R: Transformation of follicular lymphoma to diffuse large-cell lymphoma: alternative patterns with increased or decreased expression of c-myc and its regulated genes. Proc Natl Acad Sci USA 2002, 99:8886-8891

40. Freedman AS: Biology and management of histologic transformation of indolent lymphoma. Hematology Am Soc Hematol Educ Program 2005, 314-320

41. Montoto S, Davies AJ, Matthews J, Calaminici M, Norton AJ, Amess J, Vinnicombe S, Waters R, Rohatiner AZ, Lister TA: Risk and clinical implications of transformation of follicular lymphoma to diffuse large B-cell lymphoma. J Clin Oncol 2007, 25:2426-2433

42. Acker B, Hoppe RT, Colby TV, Cox RS, Kaplan HS, Rosenberg SA: Histologic conversion in the non-Hodgkin's lymphomas. J Clin Oncol 1983, 1:11-16

43. Miyake M, Nakano K, leki Y, Adachi M, Huang CL, Itoi S, Koh T, Taki $\mathrm{T}$ : Motility related protein 1 (MRP-1/CD9) expression: inverse correlation with metastases in breast cancer. Cancer Res 1995, 55:4127-4131

44. Higashiyama M, Taki T, leki Y, Adachi M, Huang CL, Koh T, Kodama K, Doi O, Miyake M: Reduced motility related protein-1 (MRP-1/CD9) gene expression as a factor of poor prognosis in non-small cell lung cancer. Cancer Res 1995, 55:6040-6044

45. Cajot JF, Sordat I, Silvestre T, Sordat B: Differential display cloning 
identifies motility-related protein (MRP1/CD9) as highly expressed in primary compared with metastatic human colon carcinoma cells. Cancer Res 1997, 57:2593-2597

46. Houle CD, Ding XY, Foley JF, Afshari CA, Barrett JC, Davis BJ: Loss of expression and altered localization of KAl1 and CD9 protein are associated with epithelial ovarian cancer progression. Gynecol Oncol 2002, 86:69-78

47. Wang JC, Begin LR, Berube NG, Chevalier S, Aprikian AG, Gourdeau $\mathrm{H}$, Chevrette M: Down-regulation of CD9 expression during prostate carcinoma progression is associated with CD9 mRNA modifications. Clin Cancer Res 2007, 13:2354-2361

48. De Bruyne E, Bos TJ, Asosingh K, Vande Broek I, Menu E, Van Valckenborgh E, Atadja P, Coiteux V, Leleu X, Thielemans K, Van Camp B, Vanderkerken K, Van Riet I: Epigenetic silencing of the tetraspanin CD9 during disease progression in multiple myeloma cells and correlation with survival. Clin Cancer Res 2008, 14:2918-2926

49. Takeda T, Hattori N, Tokuhara T, Nishimura Y, Yokoyama M, Miyake M: Adenoviral transduction of MRP-1/CD9 and KAl1/CD82 inhibits lymph node metastasis in orthotopic lung cancer model. Cancer Res 2007, 67:1744-1749

50. Ovalle S, Gutierrez-Lopez MD, Olmo N, Turnay J, Lizarbe MA, Majano P, Molina-Jimenez F, Lopez-Cabrera M, Yanez-Mo M, Sanchez-Madrid F, Cabanas C: The tetraspanin CD9 inhibits the proliferation and tumorigenicity of human colon carcinoma cells. Int J Cancer 2007 121:2140-2152

51. Zvereff V, Wang JC, Shun K, Lacoste J, Chevrette M: Colocalisation of CD9 and mortalin in CD9-induced mitotic catastrophe in human prostate cancer cells. Br J Cancer 2007, 97:941-948

52. Van Riet I, De Greef C, Aharchi F, Woischwill C, De Waele M, Bakkus M, Lacor P, Schots R, Van Camp B: Establishment and characterization of a human stroma-dependent myeloma cell line (MM5.1) and its stroma-independent variant (MM5.2). Leukemia 1997, 11:284-293

53. De Bruyne E, Andersen TL, De Raeve H, Van Valckenborgh E, Caers J, Van Camp B, Delaisse JM, Van Riet I, Vanderkerken K: Endothelial cell-driven regulation of CD9 or motility-related protein-1 expression in multiple myeloma cells within the murine 5T33MM model and myeloma patients. Leukemia 2006, 20:1870-1879

54. Masellis-Smith A, Shaw AR: CD9-regulated adhesion. Anti-CD9 monoclonal antibody induce pre-B cell adhesion to bone marrow fibroblasts through de novo recognition of fibronectin. J Immunol 1994, 152:2768-2777

55. Longo N, Yanez-Mo M, Mittelbrunn M, de la Rosa G, Munoz ML, Sanchez-Madrid F, Sanchez-Mateos P: Regulatory role of tetraspanin CD9 in tumor-endothelial cell interaction during transendothelial invasion of melanoma cells. Blood 2001, 98:3717-3726

56. Barreiro O, Yanez-Mo M, Sala-Valdes M, Gutierrez-Lopez MD, Ovalle S, Higginbottom A, Monk PN, Cabanas C, Sanchez-Madrid F: Endothelial tetraspanin microdomains regulate leukocyte firm adhesion during extravasation. Blood 2005, 105:2852-2861

57. Higashiyama S, Iwamoto R, Goishi K, Raab G, Taniguchi N, Klagsbrun M, Mekada E: The membrane protein CD9/DRAP 27 potentiates the juxtacrine growth factor activity of the membrane-anchored heparin-binding EGF-like growth factor. J Cell Biol 1995, 128:929-938

58. Nakamura K, Mitamura T, Takahashi T, Kobayashi T, Mekada E: Importance of the major extracellular domain of CD9 and the epidermal growth factor (EGF)-like domain of heparin-binding EGF-like growth factor for up-regulation of binding and activity. J Biol Chem 2000, 275:18284-18290

59. Yau JC, Dabbagh LK, Formenti KS, Coupland RW, Burns BF, Shaw AR: Expression of transmembrane 4 superfamily member, CD9, is related to improved progression-free survival in patients with diffuse non-Hodgkin's Iymphoma. Oncol Rep 1998, 5:1507-1511

60. Zhong S, Fields CR, Su N, Pan YX, Robertson KD: Pharmacologic inhibition of epigenetic modifications, coupled with gene expression profiling, reveals novel targets of aberrant DNA methylation and histone deacetylation in lung cancer. Oncogene 2007, 26:2621-2634 